\title{
The Role of Metal-Nanotube Contact in the Performance of Carbon Nanotube Field-Effect Transistors
}

2005

Vol. 5, No. 7

1497-1502

\author{
Zhihong Chen, ${ }^{\dagger}$ Joerg Appenzeller, ${ }^{*, \dagger}$ Joachim Knoch, ${ }^{\ddagger}$ Yu-ming Lin, ${ }^{\dagger}$ and \\ Phaedon Avouris ${ }^{*, \dagger}$
}

\author{
IBM T. J. Watson Research Center, Yorktown Heights, New York 10598, and Institute \\ of Thin Film and Interfaces and Center of Nanoelectronic Systems, \\ Forschungszentrum Julich, D-52454, Julich, Germany
}

Received May 9, 2005; Revised Manuscript Received June 6, 2005

\begin{abstract}
Single-wall carbon nanotube field-effect transistors (CNFETs) have been shown to behave as Schottky barrier (SB) devices. It is not clear, however, what factors control the SB size. Here we present the first statistical analysis of this issue. We show that a large data set of more than 100 devices can be consistently accounted by a model that relates the on-current of a CNFET to a tunneling barrier whose height is determined by the nanotube diameter and the nature of the source/drain metal contacts. Our study permits identification of the desired combination of tube diameter and type of metal that provides the optimum performance of a CNFET.
\end{abstract}

Since the first carbon nanotube field-effect transistor $(\mathrm{CN}$ FET $)^{1,2}$ was experimentally demonstrated about six years ago, significant progress has been made in understanding the transistor physics and improving the device performance. With exceptional electrical properties, such as quasi-ballistic transport $^{3,4}$ or high carrier mobility in the diffusive regime, ${ }^{5}$ CNFET devices nowadays exhibit characteristics rivaling those of state-of-the-art Si-based MOSFETs. Since it has been generally accepted that Si-based CMOS technology will reach its scaling limit in the next decade or so, exploration of the potential of carbon nanotubes as building blocks in future nanoelectronics is needed.

One of the most important CNFET characteristics is the current in the device on-state, referred to as the on-current, $I_{\text {on }}$. Reproducibility in the device current is essential in particular from the point of view of circuit applications. However, CNFETs using similar device geometries reported in the literature deliver on-currents ranging from $10^{-5}$ to $10^{-8}$ A. While different nanotube sources and metal contacts can be expected to be responsible for the observed range of $I_{\mathrm{on}}$, a thorough comparison has not been performed and a clear conclusion about the origin of variation has not yet been drawn. ${ }^{6}$

In this paper, we report an investigation of over a hundred CNFETs using three different source/drain (S/D) metal

\footnotetext{
* Corresponding authors. E-mail: joerga@us.ibm.com; avouris@ us.ibm.com.

IBM T. J. Watson Research Center.

Institute of Thin Film and Interfaces and Center of Nanoelectronic Systems.
}

contacts and nanotubes with fairly large diameter variations. The dependence of the on-current in a CNFET on the nanotube diameter and the nature of the metal contact is studied. We find that a model that relates the on-current to a Schottky barrier (SB) height determined by the nanotube diameter and metal contact can account for the large set of data including those from other groups. One objective of this study is to identify the desired combination of nanotube diameter and type of metal that allows reproducibility for optimum CNFET device performance.

CNFETs studied here are fabricated using a standard backgate geometry, as shown in the inset of Figure 1. Laser ablation grown carbon nanotubes from Rice University ${ }^{7}$ are suspended in 1,2-dichloroethane solution by sonication and then spun onto p-doped Si substrates covered by $10 \mathrm{~nm}$ of thermally grown $\mathrm{SiO}_{2}$. A $\mathrm{Si}$ substrate is used as the back gate with the $\mathrm{SiO}_{2}$ as the gate dielectric. Source (S) and drain (D) contact patterns with a spacing of $300 \mathrm{~nm}$ are defined by e-beam lithography. Three metals, palladium (Pd), titanium (Ti), and aluminum (Al), are used as S/D contacts. All metal contacts are e-beam evaporated in the same vacuum system at a base pressure of $10^{-8}$ Torr followed by a standard lift-off process. The measurements are carried out under nitrogen flow with an HP semiconductor analyzer.

Figure 1a shows the drain current $I_{\mathrm{d}}$ as a function of the gate voltage $V_{\mathrm{gs}}$ for three Pd-contacted CNFETs using three different nanotubes at a drain voltage of $V_{\mathrm{ds}}=-0.5 \mathrm{~V}$. All three devices are in the on-state when $V_{\mathrm{gs}} \ll 0 \mathrm{~V}$, which indicates hole carrier injection from the source metal contact 


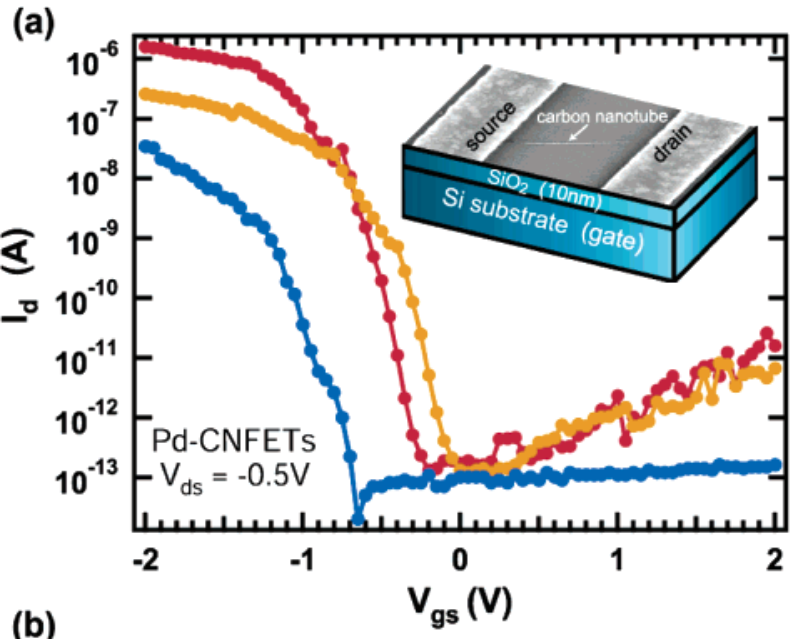

(b)

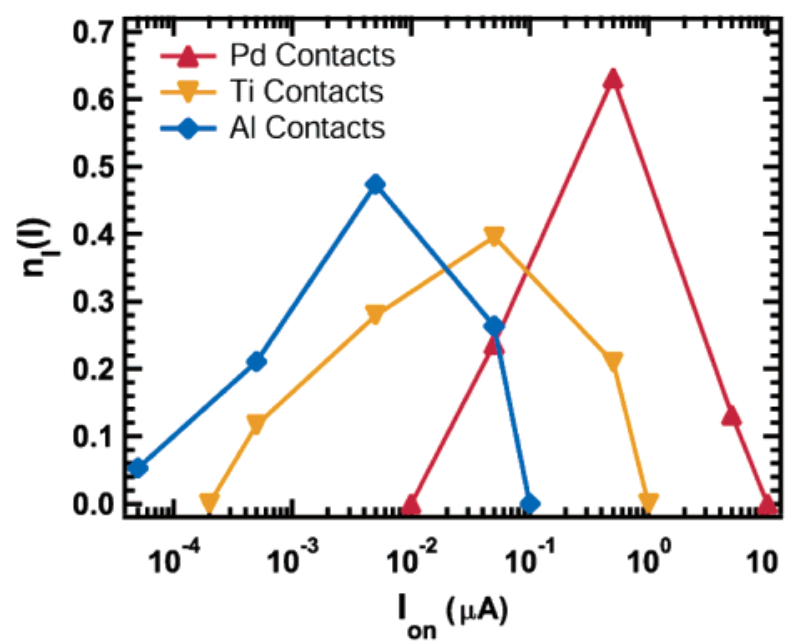

(C)

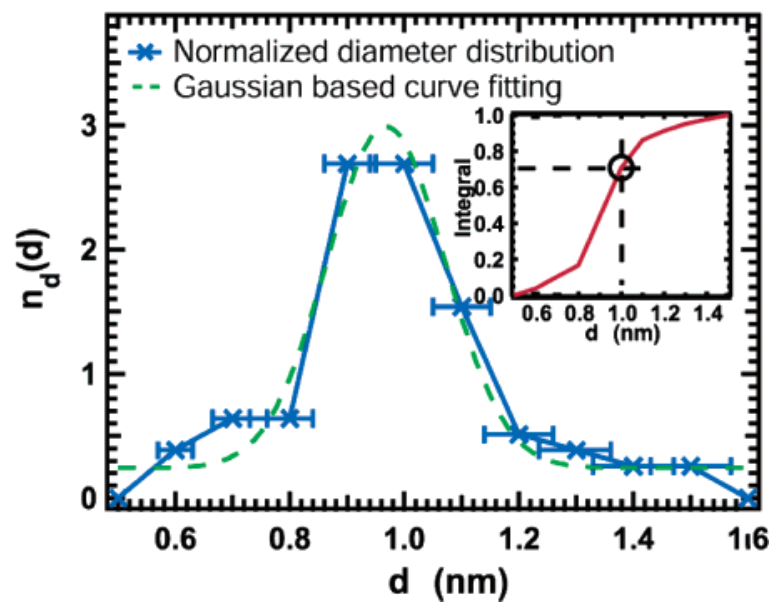

Figure 1. (a) Subthreshold characteristics of three Pd contacted CNFET devices at $V_{\mathrm{ds}}=-0.5$ V. $I_{\mathrm{on}}$ is defined at $V_{\mathrm{gs}}-V_{\mathrm{th}}=$ $-0.5 \mathrm{~V}$. Inset shows a SEM image of a carbon nanotube fieldeffect transistor with $300 \mathrm{~nm}$ channel length. (b) Normalized oncurrent distribution, $n_{I}$, for $38 \mathrm{Pd}-\mathrm{CNFETs}, 43 \mathrm{Ti}-\mathrm{CNFETs}$, and $25 \mathrm{Al}-$ CNFETs. (c) Normalized diameter distribution, $n_{d}$, for 78 nanotubes (blue solid line), with a Gaussian function curve fitting (green dotted line). Inset shows $\int_{d_{0}}^{d} n_{d} \mathrm{~d} x$ as a function of $d$.

into the valence band of the nanotube. We will focus our discussion on this so-called p-type branch of the characteristics unless otherwise stated. The most important aspect to note is that an on-current variation of approximately 2 orders of magnitude is observed among these three devices, although the same metal contacts and identical fabrication processes were employed.

We fabricated and studied $38 \mathrm{Pd}$ contacted CNFETs ( $\mathrm{Pd}-$ CNFETs), 43 Ti contacted CNFETs (Ti-CNFETs) and 25 $\mathrm{Al}$ contacted CNFETs (Al-CNFETs) on three sample chips. $I_{\mathrm{on}}$ is recorded for every device at $V_{\mathrm{ds}}=V_{\mathrm{gs}}-V_{\mathrm{th}}=-0.5$ $\mathrm{V}$ with $V_{\text {th }}$ being the threshold voltage. ${ }^{8}$ This fairly large drain voltage helps overcome possible small barriers associated with contamination at the metal/nanotube interface and ensures that we are indeed characterizing the intrinsic device properties. The choice of defining $I_{\mathrm{on}}$ at $V_{\mathrm{gs}}-V_{\mathrm{th}}=-0.5 \mathrm{~V}$ also reduces the systematic error that may occur from the uncertainty in determining the threshold voltage. Large current variation is observed among these devices. The oncurrent distributions $n_{I}\left(I_{\text {on }}\right)$ for the three contact metals are shown in Figure 1b, in which the number of devices, normalized by the total device number in each metal sample, is displayed as a function of $I_{\mathrm{on}}$. A similar triangular distribution and around 3-4 orders of magnitude current variation are observed for all three types of metal contacts. The common current distribution shape suggests a common variation source existing for all three samples.

In our CNFET devices, S/D metals are in direct contact with the nanotubes. At a metal/semiconductor interface, a barrier is formed whose height depends on the line-up between the metal Fermi level and the valence/conduction band of the semiconductor. In general, it is more difficult to make a "good" contact to a wide-gap semiconductor since usually large barriers occur at the interface. ${ }^{8}$ If CNFETs are fabricated using semiconducting nanotubes with different energy gaps, $E_{\mathrm{g}}$, current variation can be expected among these devices as a result of an $E_{\mathrm{g}}$-dependent line-up between the metal Fermi level and the conduction/valence band of the semiconductor. Since $E_{\mathrm{g}}$ depends inversely on the nanotube diameter, ${ }^{9} d$, measuring $d$ is a means to obtain information about $E_{\mathrm{g}}$.

An initial attempt to correlate the CNFET on-current with the nanotube diameter forming the CNFET has been made by measuring the nanotube height from atomic force microscopy (AFM) images. ${ }^{10}$ However, the difference in nanotube diameters is often on the scale of angstroms, beyond the accuracy of the AFM measurement. Our approach is to make transmission electron microscopy (TEM) measurements on a sample containing nanotubes from the same source used for the CNFET devices. TEM focus variation tests and measurements along the length of a nanotube show less than $\pm 5 \%$ systematic errors when determining the nanotube diameter. By characterizing a large number of tubes, we are able to provide statistical information on the nanotube diameters. Figure 1c plots the normalized diameter distribution, $n_{\mathrm{d}}$, for 78 randomly selected nanotubes, along with a Gaussian function curve fitting, showing that the batch of laser ablation nanotubes used here consists of nanotubes possessing diameters between 0.6 and $1.5 \mathrm{~nm}$ with the distribution centered around $1 \mathrm{~nm}$. From tight binding 
calculations, one can infer a corresponding variation of energy gaps between 0.6 and $1.4 \mathrm{eV}$ (using an overlap integral of $|t|=3 \mathrm{eV}),{ }^{9}$ and we will argue in the following that it is this gap energy variation that can consistently explain our measurements.

With enough statistics in both diameter and device measurements, we can correlate the device on-currents with the nanotube diameters by comparing the integral of each distribution. The inset of Figure 1c shows the integral for the diameter distribution, where the circled point implies that $70 \%$ of the nanotubes have diameters smaller than $1 \mathrm{~nm}$. Similar integral curves are obtained for the current distributions. By comparing the integral values of the two distributions for 11 selected currents, we assign a nanotube diameter to a particular $I_{\text {on }}$ value: $\int_{d_{0}}^{d} n_{d} \mathrm{~d} x=\int_{I_{0}}^{I_{\mathrm{on}}} n_{I} \mathrm{~d} x \rightarrow I_{\mathrm{on}}(d)$, where $d_{0}$ is the smallest diameter and $I_{0}$ is the lowest oncurrent measured. Figure 1 summarizes the diameter dependence of $I_{\text {on }}$ for Pd-, Ti-, and Al-contacted CNFETs. The error bars in the graph account for the uncertainty in $n_{I}$ due to the inability to accurately determine $I_{\mathrm{on}}$. This uncertainty becomes less pronounced at the distribution peak because of the large number of devices evaluated for the corresponding current levels. Therefore, devices with average diameter nanotubes have smaller error bars. ${ }^{11}$ Similar trends for $I_{\text {on }}$ are observed in all three metal contacted samples. For any nanotube diameter, p-type Pd-CNFETs have the highest $I_{\text {on }}$ and $\mathrm{Al}$-CNFETs have the lowest $I_{\mathrm{on}}$. While this trend follows that of the clean metal work functions: $5.1 \mathrm{eV}$ for $\mathrm{Pd}, 4.3 \mathrm{eV}$ for $\mathrm{Ti}$, and $4.1 \mathrm{eV}$ for $\mathrm{Al}$, the values of $I_{\mathrm{on}}$ are not directly correlated with the macroscopic clean surface work function values. We note that while $I_{\text {on }}$ increases by the same amount from $\mathrm{Al}$ to $\mathrm{Ti}$ as from $\mathrm{Ti}$ to $\mathrm{Pd}$ on the log scale, the difference in work functions is much larger between $\mathrm{Pd}$ and $\mathrm{Ti}$ than between $\mathrm{Ti}$ and $\mathrm{Al}$. Another important information obtained from this graph is that $I_{\mathrm{on}}$ exhibits a larger variation for small nanotubes than for large diameter nanotubes.

To confirm that the local contact quality is not the major contribution to the observed current variation, we also fabricated serval CNFETs on the same long nanotube. A factor of 2 current variation was observed among devices along the same tube, contrary to the 3-4 orders of magnitude current variation shown above. To further prove the general validity of the diameter dependence of the CNFET oncurrent, and show that it is independent of the nanotube source, we have added data points from other publications, as shown in the inset of Figure 1. Data point "1" represents CNFETs using arc discharge grown nanotubes. ${ }^{12}$ Devices using these nanotubes have very similar $I_{\mathrm{on}}$, which is consistent with our finding from TEM measurements that the arc discharge tubes exhibit an average diameter of 1.8 $\pm 0.2 \mathrm{~nm}$. For data points " 2 " and "3", CVD grown nanotubes were used, and the diameters were estimated by AFM measurements. ${ }^{13,14}$ A CNFET with a channel length of $300 \mathrm{~nm}$ has essentially the same electrical behavior as one with a $50 \mathrm{~nm}$ channel length due to the quasi-ballistic transport in the nanotubes, and therefore, the data point " 2 " of the CVD grown nanotube devices with same gate oxide

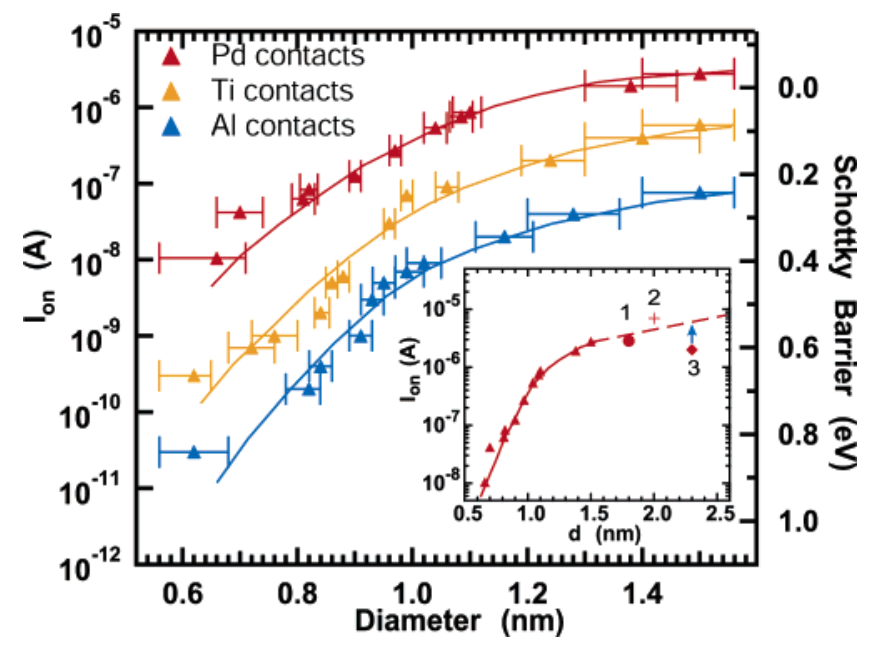

Figure 2. Plot of CNFETs $I_{\mathrm{on}}$ as a function of nanotube diameter, $d$, for $\mathrm{Pd}, \mathrm{Ti}$, and $\mathrm{Al}$ metal contacts. Devices are fabricated on 10 $\mathrm{nm} \mathrm{SiO}$, with channel length of $300 \mathrm{~nm} . V_{\mathrm{ds}}=-0.5 \mathrm{~V}$ is applied to all devices, and $I_{\mathrm{on}}$ is defined at $V_{\mathrm{gs}}-V_{\mathrm{th}}=-0.5 \mathrm{~V}$. The right axis is the $\phi_{\mathrm{SB}}$ extracted from $I_{\mathrm{on}}$ using the extended SB model. Inset includes data points for Pd contacted CNFETs from other publications. All data points are taken at $V_{\mathrm{DD}}=-0.5 \mathrm{~V}$. Point 1 : diameter $d=1.8 \mathrm{~nm}, 10 \mathrm{~nm} \mathrm{SiO}{ }_{2}$, channel length $L=300 \mathrm{~nm} \cdot{ }^{12}$ Point 2: $d=2.0 \mathrm{~nm}, 10 \mathrm{~nm} \mathrm{SiO}_{2}, L=50 \mathrm{~nm} .{ }^{13}$ Point 3: $d=2.3$ $\mathrm{nm}, 10 \mathrm{~nm} \mathrm{SiO}{ }_{2}, L=2 \mu \mathrm{m} .{ }^{14}$

but $50 \mathrm{~nm}$ channel length fits well into our curve. Point " 3 " has a lower current than that predicted because its over 2 $\mathrm{mm}$ long channel allows for acoustic phonon scattering. Overall, the current dependence on the nanotube diameter follows the trend that our graph predicts independent of the nanotube source.

CNFETs have been found to be SB devices. ${ }^{15-17}$ At the metal/nanotube interface, hole injection into the nanotube is dependent on the line-up of the metal Fermi level and the valence band of the nanotube, which is defined here as the SB height, $\phi_{\mathrm{SB}}$. In this picture, other details of the contacts such as any changes in the metal-nanotube coupling as a function of the curvature of the nanotube are incorporated in an "effective" SB tunneling barrier height. Figure 2a shows qualitative band diagrams for CNFETs with different diameters. Assuming a constant work function ${ }^{18}$ for all nanotubes, $\phi_{\mathrm{SB}}$ increases linearly with increasing $E_{\mathrm{g}}$. On a $\log$ scale, current injection through the SB is inversely proportional to the barrier height; therefore, the CNFET with a small diameter $\left(E_{\mathrm{g}-} C\right)$ delivers the lowest on-current. The choice of the metal contacts also affects the device performance. Figure $2 \mathrm{~b}$ depicts the band diagrams for CNFETs using different S/D contacts; identical energy gaps are drawn here to represent the same diameter nanotubes. Since Pd has the highest work function, which forms a low $\phi_{\mathrm{SB}}$ to the valence band of the nanotube, Pd-CNFETs deliver the highest $I_{\mathrm{on}}$. The observed trend in the experiments is consistent with the expected dependence of $I_{\mathrm{on}}$ on the nanotube diameter and metal contacts.

Given the current dependence on the SB height and the linear dependence between $\phi_{\mathrm{SB}}$ and $E_{\mathrm{g}}$, one can expect a linear response of the $\log \left(I_{\mathrm{on}}\right)$ on the inverse of the tube diameter: $\log \left(I_{\text {on }}\right) \propto-E_{\mathrm{g}} \propto-1 / d$. We plot $I_{\text {on }}$ in log scale 


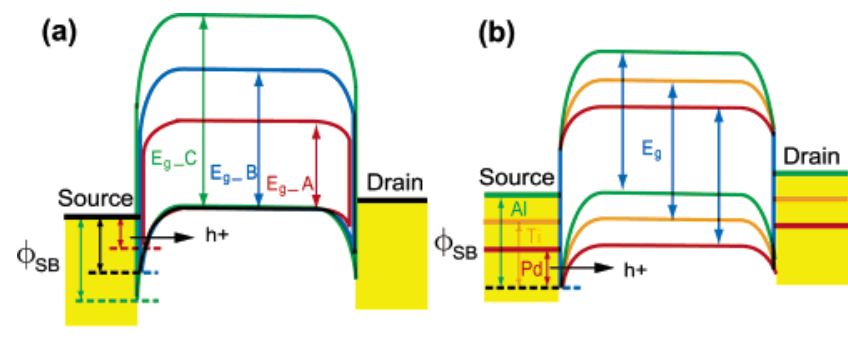

(c)

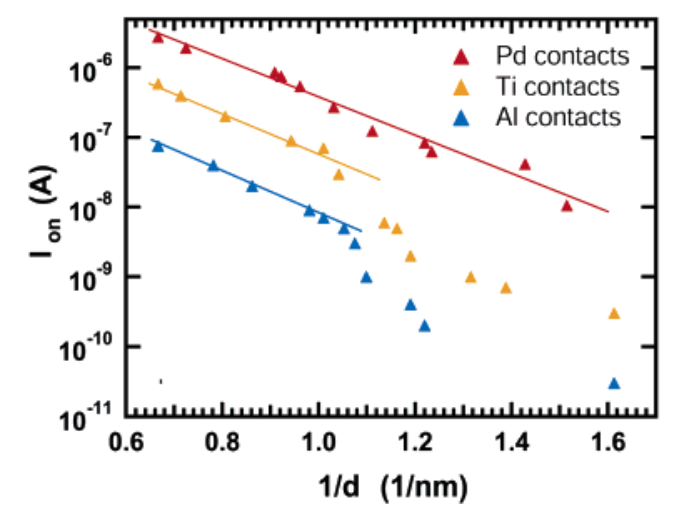

Figure 3. (a) Schematic band diagram depicts the Schottky barrier height differences in three same contacts CNFETs using nanotubes with different diameters. (b) Schematic band diagram depicts the $\phi_{\mathrm{SB}}$ differences in three CNFETs with same diameter nanotube, but using $\mathrm{Pd}$, Ti, and Al contacts, respectively. (c) log scale oncurrent as a function of $1 / d$ for $\mathrm{Pd}, \mathrm{Ti}$, and $\mathrm{Al}$ contacted CNFETs, generated from Figure 1.

as a function of $1 / d$ in Figure 3c, with solid lines indicating the linear response. All Pd-CNFETs and Ti- as well as Alcontacted devices with large diameter nanotubes clearly follow the anticipated trend. Some deviation from the expected response is visible from small diameter nanotubesan observation that may be related to the more reactive nature of Ti and Al. The common slope for all three metal contact samples clearly supports our interpretation of the current dependence on the nanotube diameter, and the metal work function difference explains the offsets among the three curves, though the offset values are not directly correlated with the macroscopic clean metal work function values as mentioned earlier.

So far, we have argued that the dependence of current on tube diameter and the nature of metal is predominating a contact effect which implies quasi-ballistic transport through the nanotube channel. Shorter devices $(40 \mathrm{~nm})$ with different metal contacts have been fabricated and show a similar current behavior as the $300 \mathrm{~nm}$ devices investigated in this study. Therefore, we conclude that the channel length of 300 $\mathrm{nm}$ used in our devices is short enough to ignore scattering by acoustic phonons, ${ }^{19-21}$ but we still need to evaluate whether optical phonon scattering impacts our previous statements under the drain voltage and overdrive conditions applied. According to a recent calculation, ${ }^{21}$ optical phonon scattering is more severe for small diameter tubes than for larger diameter ones. However, even for a diffusive channel the difference in scattering for different tube diameters is expected to result in $0.1-0.2$ decade of current variation for the voltages applied in our devices. ${ }^{21}$ Therefore, the 3-4 orders of magnitude current variation for samples using same metal contacts cannot be a result of the scattering difference. To explore further this issue, we performed measurements for Pd-CNFETs at a lower drain voltage of $V_{\mathrm{ds}}=-0.1 \mathrm{~V}$ as well, and have recorded $I_{\text {on }}$ at $V_{\mathrm{gs}}-V_{\text {th }}=-0.1 \mathrm{~V}$ (define $\left.V_{\mathrm{DD}}=V_{\mathrm{ds}}=V_{\mathrm{gs}}-V_{\mathrm{th}}\right)$. At this voltage, optical phonon scattering is not allowed since $\hbar \omega_{\mathrm{ph}} \sim 0.18 \mathrm{eV}$. We find that the behavior of the current trend is similar for both voltages which suggests that optical phonon scattering does not have a major impact on our analysis even for $V_{\mathrm{DD}}=$ $-0.5 \mathrm{~V}$. This confirms that in our experiments the diameter of the nanotube is the key parameter in determining the CNFET on-current for a given metal contact.

In the following, we introduce an extended SB model for CNFETs in order to compare our experimental results with simulations and extract the effective SB heights of our devices. While existing SB models ${ }^{15-17,22}$ capture some scaling aspects of the nanotube device performance, a closer look at simulated and experimental data reveals a number of discrepancies. In particular, simple SB models do not explain the often observed highly asymmetric $\mathrm{I}_{\mathrm{d}}\left(V_{\mathrm{gs}}\right)$ curves with substantially smaller currents for positive than for negative gate voltages even when a thin gate dielectric is used. ${ }^{23}$

Simple models fail to capture certain aspects observed in the experiment since the contact geometry of a "real" CNFET is not adequately described by the orifice-type contact between the metal and the nanotube usually assumed. Indeed, the real contact between the metal electrode and the nanotube is made over an overlap region that extends for several hundred nanometers. To describe this situation properly, we introduce an extended SB model. Tersoff and others ${ }^{24,25}$ have pointed out that a substantial potential barrier exists between the nanotube and the metal contact in the overlap region. Our extended model takes this tunneling barrier phenomenologically into account and describes the CNFET as a gated nanotube channel in contact with two reservoirs that are a result of the weak coupling between the metal and the nanotube in the overlap region.

To model the situation in a CNFET, we self-consistently solve the Poisson and Schrödinger equation using the nonequilibrium Green's function formalism (NEGF) on a finite difference grid. A modified, one-dimensional Poisson equation is well suited to describe the electrostatics of CNFETs. ${ }^{26}$ The following assumptions are made in our calculations: (i) A quadratic dispersion relation with equal effective masses in the conduction and valence bands is assumed; an energy dependent effective mass takes the complex band structure in the band gap into account. ${ }^{26}$ (ii) The contact metals are considered as ideal conductors with a quadratic dispersion and free electron effective mass. (iii) Metal contact and the nanotube underneath are in equilibrium with the same Fermi level. (iv) Finally, ballistic transport is considered. Within the NEGF, the metal attached to the nanotube is accounted for by an appropriate self-energy function $^{27}$ at each grid point in the metal/nanotube contact region. To describe a coupling of varying strength, the self- 


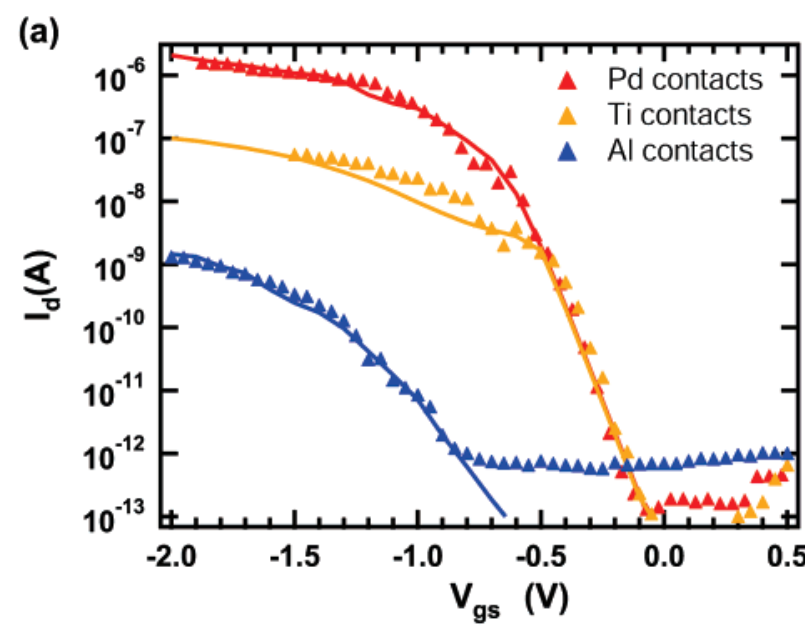

(b)

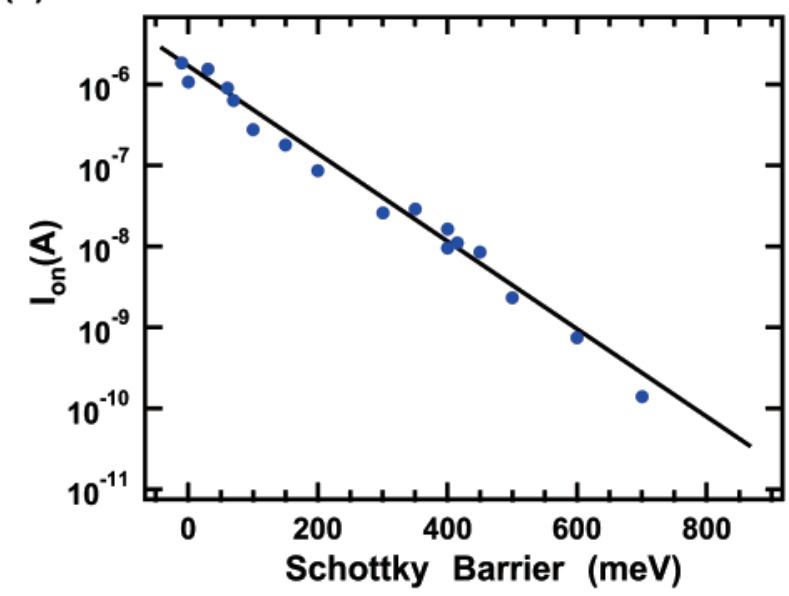

Figure 4. (a) Measured and simulated characteristics for three CNFETs with Pd, Ti, and Al contacts, respectively. (b) Schottky barrier and $I_{\mathrm{on}}$ calibration curve. Extended SB model with two fitting parameters, the coupling strength $\gamma$ and the SB height, is used to fit a set of experimental characteristics. With the same coupling strength constant, the current and SB calibration curve is obtained.

energy is multiplied by a factor $\gamma=0.0 .1$, where $\gamma=0$ implies no coupling and $\gamma=1$ refers to perfect coupling. It can be shown that $\gamma$ can be related to a potential barrier of a certain height and width present at the metal-nanotube interface. This is done by calculating the transmission probability through all grid sites coupled to two contacts where the coupling of one contact is mediated by the parameter $\gamma$ and comparing this transmission probability with the transmission probability through a potential barrier. For a barrier of $\gtrsim 10 \mathrm{eV}$ and a distance between metal and nanotube $\gtrsim 3 \AA, \gamma$ follows to be less than 0.01 (for more details see ref 23 ).

Three representative characteristics selected from three metal samples are shown in Figure $4 \mathrm{a}$ along with the simulation results. A coupling constant of $\gamma=0.007$ gives good agreement between simulation and experiment, in both the off- and the on-state. The only adjustable parameter used for the three devices is the effective $\phi_{\mathrm{SB}}$. Note that the relative shift of $V_{\text {th }}$ seen in the simulations is a consequence of a varying $\phi_{\mathrm{SB}}$ and this shift matches the experimental data very well. Using this approach, we have simulated electrical characteristics for a wide range of $\phi_{\mathrm{SB}}$ values. Figure $4 \mathrm{~b}$ shows the exponential dependence of the on-current on the barrier height. Using Figure $4 \mathrm{~b}$ as a calibration, we add a right axis to Figure 1 giving the $\phi_{\mathrm{SB}}$ as a function of diameter and in this way providing a more general, i.e., gate oxide thickness and drain/gate voltage independent, correlation between nanotube diameter and Fermi level line up. So far there have been only a few attempts to extract quantitative values for $\phi_{\mathrm{SB}}$ from experiments on CNFETs. However, the extracted $\phi_{\mathrm{SB}}$ of $\sim 360 \mathrm{meV}$ for Ti-CNFETs with a nanotube diameter of $d \approx 1 \mathrm{~nm}^{28}$ and a barrier close to zero for a $\mathrm{Pd}-\mathrm{CNFET}$ with $d \approx 1.7 \mathrm{~nm}^{29}$ are in reasonable agreement with our current study on nanotube contacts.

In summary, we have investigated over a hundred CNFETs using nanotubes with various diameters and different metal contacts. We find that the variation in nanotube diameter is mainly responsible for the observed large current variation from device to device in our CNFETs. Our own data as well as experimental results from other groups can be consistently explained as the result of the particular choice of nanotube and metal contact type. According to our findings, the best $\mathrm{p}$-CNFET on-state performance (with small current variation for CNFETs having slightly different diameters) can be achieved by combining CNTs with diameters above $1.4 \mathrm{~nm}$ with Pd contacts. An extended SB model is used to extract the effective SB height at the contact/nanotube interface to yield a comprehensive picture of the band line up in CNFETs of various type of nanotubes and contact metals.

Acknowledgment. The authors thank Dr. Ruud M. Tromp and Dr. Christian Klinke for valuable discussions on the accuracy of TEM measurements and Bruce Ek for metal deposition and expert technical assistance.

\section{References}

(1) Tans, S. J.; Verschueren, A. R. M.; Dekker, C. Nature 1998, 393 , 49.

(2) Martel, R.; Schmidt, T.; Shea, H. R.; Hertel, T.; Avouris, Ph. Appl. Phys. Lett. 1998, 73, 2447.

(3) Wind, S.; Appenzeller, J.; Avouris, Ph. Phys. Rev. Lett. 2003, 91, 58301.

(4) Javey, A.; Guo, J.; Paulsson, M.; Wang, Q.; Mann, D.; Lundstrom, M.; Dai, H. Phys. Rev. Lett. 2004, 92, 106804.

(5) Durkop, T.; Getty, S. A.; Cobas, E.; Fuhrer, M. S. Nano Lett. 2004, 4,35 .

(6) It has been pointed out before qualitatively ${ }^{10}$ that a monotonic trend between $I_{\mathrm{on}}$ and tube diameter exists for Pd-contacted CNFETs.

(7) Thess, A.; Lee, R.; Nikolaev, P.; Dai, H. J.; Petit, P.; Robert, J.; $\mathrm{Xu}$, C. H.; Lee, Y. H.; Kim, S. G.; Rinzler, A. G.; et al. Science 1996, 273, 483.

(8) Sze, S. Physics of semicondutor devices, 2nd ed.; John Wiley and Sons: New York, 1981.

(9) Dresselhaus, M. S.; Dresselhaus, G.; Avouris, Ph. Carbon nanotubes: synthesis, structure, properties, and applications; Springer-Verlag: Berlin, 2001.

(10) Javey, A.; Guo, J.; Wang, Q.; Lundstrom, M.; Dai, H. Nature 2003, $424,654$.

(11) The $\pm 5 \%$ error found from TEM measurements is included in Figure $1 \mathrm{c}$ and is used to determine $n_{d}(d)$. Once $n_{d}(d)$ is found, we can correlate $n_{I}(I)$ and $n_{d}(d)$. The $x$ axis in Figure 2 is the average $d$ determined from this correlation, but the error bars in the graph do not include the uncertainty in evaluating $d$ from TEM measurements, which should be taken into consideration when using Figure 2

(12) Lin, Y.-M.; Appenzeller, J.; Chen, Z.; Chen, Z.-G.; Cheng, H.-M.; Avouris, Ph. Submitted for publication in IEEE Electron Device Lett.

(13) Javey, A.; Qi, P.; Wang, Q.; Dai, H. Proc. Natl. Acad. Sci. U. S. A 2004, 101, 13408 
(14) Javey, A.; Guo, J.; Farmer, D. B.; Wang, Q.; Wang, D.; Gordon, R. G.; Lundstrom, M.; Dai, H. Nano Lett. 2004, 4, 447.

(15) Martel, R.; Derycke, V.; Lavoie, C.; Appenzeller, J.; Chan, K.; Terso, J.; Avouris, Ph. Phys. Rev. Lett. 2001, 87, 256805.

(16) Appenzeller, J.; Knoch, J.; Derycke, V.; Martel, R.; Wind, S.; Avouris, Ph. Phys. Rev. Lett. 2002, 89, 126801.

(17) Heinze, S.; Terso, J.; Martel, R.; Derycke, V.; Appenzeller, J.; Avouris, Ph. Phys. Rev. Lett. 2002, 89, 106801.

(18) The work function is defined as the sum of the nanotube electron affinity and half of the band gap in the bulk.

(19) Yao, Z.; Kane, C. L.; Dekker, C. Phys. Rev. Lett. 2000, 84, 2941.

(20) Park, J.-Y.; Rosenblatt, S.; Yaish, Y.; Sazonova, V.; Ustunel, H.; Braig, S.; Arias, T. A.; Brouwer, P. W.; MuEuen, P. L. Nano Lett. 2004, 4, 517

(21) Perebeinos, V.; Terso, J.; Avouris, Ph. Phys. Rev. Lett. 2005, 94, 86802 .

(22) Guo, J.; Datta, S.; Lundstrom, M. IEEE Trans. Electron Devices 2004, 51, 172 .
(23) Knoch, J.; Mantl, S.; Lin, Y.; Chen, Z.; Avouris, Ph.; Appenzeller, J. Device Research Conference; TMS: Warrendale, PA, 2004; pp 135-136. ISBN 0-7803-8284.

(24) Terso, J. Appl. Phys. Lett. 1999, 74, 2122.

(25) Rochefort, A.; Ventra, M. D.; Avouris, Ph. Appl. Phys. Lett. 2001, $78,2521$.

(26) Knoch, J.; Appenzeller, J. Carbon nanotube feld-effect transistorsthe importance of being small. in Hardware Drivers for Ambient Intelligence; Kluwer Publishing Services, 2005.

(27) Datta, S. Electronic transport in mesoscopic systems; Cambridge University Press: Cambridge, U.K., 1998.

(28) Appenzeller, J.; Radosavljevic, M.; Knoch, J.; Avouris, Ph. Phys. Rev. Lett. 2004, 92, 48301.

(29) Guo, J.; Javey, A.; Dai, H.; Lundstrom, M. International Electron Devices Meeting; IEEE: Piscataway, NJ, 2004; p 703.

NL0508624 